Volume 1

Issue 1 -- Melanoma Research

\title{
Primary Care for Melanoma: Should We Be Screaming for Screening?
}

Dennis J. Baumgardner

Alexandria Rogers

Follow this and additional works at: https://aurora.org/jpcrr

Part of the Oncology Commons

\section{Recommended Citation}

Baumgardner DJ, Rogers A. Primary care for melanoma: should we be screaming for screening?. J Patient Cent Res Rev. 2014;1:33-40. doi: $10.17294 / 2330-0698.1009$ 


\section{Primary Care for Melanoma: Should We Be Screaming for Screening?}

Dennis J. Baumgardner, MD, ${ }^{1}$ Alexandria Rogers, $\mathrm{BA}^{2}$

${ }^{1}$ Department of Family Medicine, Aurora UW Medical Group and Center for Urban Population Health, Aurora

Health Care, Milwaukee, WI; and ${ }^{2}$ Aurora Research Institute, Aurora Health Care, Milwaukee, WI

\section{Abstract}

The incidence of cutaneous malignant melanoma continues to rise in the United States. This deadly disease is potentially curable if caught at an early stage, however screening programs remain controversial. The United States Preventive Services Task Force cites insufficient evidence to recommend screening, by total-body skin examination (TBSE), for early detection of cutaneous melanoma. While definitive studies may be cost-prohibitive in the United States, more recent evidence suggests that organized programs to increase TBSE reduce mortality from melanoma. The positive impact of TBSE, and education regarding risk reduction and skin selfexamination, is most likely to be cost-effective in high-risk patients such as middle-aged and older men. This population also includes those with changing moles or those who always or usually sunburn; those with melanoma in a first-degree relative, or dysplastic nevi or extensive moles; and those with high-risk ultraviolet (UV) exposure or other risk factors. The role of new technology, such as in-office and in-home dermoscopy, continues to evolve. Primary care clinicians are challenged in everyday practice to appropriately prioritize TBSE and empower their patients for "skin awareness" and self-detection of melanoma. (J Patient-Centered Res Rev. 2014;1(1):33-40.)

\section{Keywords}

melanoma, primary health care, mass screening, primary prevention

\section{Introduction}

Cutaneous malignant melanoma is responsible for $75 \%$ of all skin cancer deaths, yet represents only $3-5 \%$ of all skin cancers. ${ }^{1}$ Each year, over 75,000 cases of melanoma are diagnosed in the United States, and approximately 9,000 individuals will die of this disease. The incidence of melanoma has been on the rise for decades, and the annual incidence rate has increased over 15 -fold since the 1930s..$^{2-5}$

Correspondence: Dennis J. Baumgardner, MD

1020 N. 12th St., Suite 4180, Milwaukee, WI 53233

Phone: 414-219-5191, Fax: 414-219-3116,

Email: dennis.baumgardner@aurora.org
Table 1. Melanoma Incidence Facts

Overall lifetime risk in USA is $2 \%^{3}$

$2 \%$ of all melanoma cases occur in those $<20$ years old ${ }^{1}$

Overall, incidence and mortality rates are greater in men than women ${ }^{3}$

Between ages of 15 and 39, increase in incidence has been greater in women ${ }^{3}$

Most common cause of cancer in women ages 25-29; second only to breast cancer in women ages $30-34^{1}$

Incidence rates in white persons are 7-25 times higher than persons of non-white race ${ }^{1,3}$

Of race/gender groups, highest (and increasing) incidence rates are in middle-aged and older white males ${ }^{1-5}$

Table 1 provides additional detail regarding melanoma incidence.

Overall, melanoma is most likely to occur in women on the lower extremities. In men, the trunk is the most common site of involvement; followed by the upper extremities. ${ }^{3}$ Reasons for these differences are unclear.

\section{Etiology and Risk Factors}

The etiology of the malignant transformation of melanocytes which leads to melanoma is multi-factorial and not fully understood. It includes ultraviolet (UV) radiation and genetic predisposition. ${ }^{1}$ The p53 suppressor gene is often mutated in melanoma. It is directly affected by UV radiation, especially UV-B. Solar UV exposure directly damages cells and molecules, including DNA, proteins and lipids. This leads to photoaging, immunosuppression and photocarcinogenesis. ${ }^{5}$

Risk factors for melanoma are summarized in Table 2. Melanoma risk is increased with the use of UV-emitting tanning devices, especially before age 35.,5 The recent overall increase in skin cancer in younger women may be secondary to indoor tanning. ${ }^{3}$ Interestingly, one survey of 
Table 2. Melanoma Risk Factors ${ }^{1,3,6}$

\section{Older age}

Fair skin and hair

Being more likely to sunburn rather than tan

Greater than 20 moles

Freckling

Three or more atypical moles

Immunosuppression

Previous psoralen Ultraviolet-A (PUVA) treatment

Previous squamous cell carcinoma

Solar keratoses

Xeroderma pigmentosum

A family history of melanoma (particularly first-degree relatives) or dysplastic nevi

Other significant UV exposures

inner city Brooklyn, New York, college students (41\% white) found an association between watching reality television beauty shows and outdoor tanning and tanning lamp use. ${ }^{7}$ In the United States, age-adjusted rates of early- and latestage melanoma are both significantly higher in counties with high solar UV exposure than in those with lower solar UV exposure. ${ }^{8}$ Similarly, in Australia, the country with the highest melanoma incidence rates (twice that of the United States), ${ }^{3}$ melanoma incidence rates are highest in the northern, more tropical areas of the continent. ${ }^{9}$

Higher socioeconomic status has been associated with increased melanoma incidence but decreased mortality rates among those with melanoma. ${ }^{2}$ In the United States and other developed countries, the increased incidence in those of higher socioeconomic status appears to be related to the higher incidence of Northern European ancestry within this section of society (fair hair and skin, likelihood to burn, rather than tan, increased freckling and atypical moles), and greater leisure-time sun exposure, rather than income. ${ }^{3,5}$ Females in this group are more likely to engage in indoor tanning activities than those of lower socioeconomic status. Lower socioeconomic status is related to having a more advanced stage of melanoma at the time of diagnosis, as is being uninsured or on Medicaid, as opposed to private insurance. ${ }^{3}$ Among non-Hispanic whites, declines in melanoma mortality rates in recent decades have largely occurred in the more educated groups of individuals. ${ }^{10}$

\section{The Screening Controversy}

In 2001, the United States Preventive Services Task Force (USPSTF) concluded that there was insufficient evidence to recommend for or against screening, by total-body skin examination (TBSE) for early detection of cutaneous melanoma, or squamous cell or basal cell carcinoma. ${ }^{11} \mathrm{~A}$ 2009 update of this recommendation acknowledged gaps in the relevant research literature, but again concluded that direct evidence to support the benefits of physician or patient screening skin examinations to reduce morbidity or mortality from skin cancer was lacking. ${ }^{12}$ This occurred despite the authors acknowledging that physician-discovered melanoma was at an earlier stage (thinner) than non-physician detection (see below), and limited evidence from one study ${ }^{13}$ of reduction of melanoma morbidity and mortality by skin selfexamination (SSE).

The American Cancer Society (ACS), however, endorses TBSE and monthly SSE. In addition, many researchers and clinicians are uncomfortable with the notion of not recommending some form of screening for the early detection of melanoma. Curiel-Lewandrowski and colleagues emphasize that there are several recent studies which support the potential benefit of screening for melanoma. ${ }^{14}$ Mitchell and Leslie opine that "we have failed to make significant gains in mortality (from melanoma) during the past 20 years because we have not empowered our society with the basic, crucial knowledge to detect disease earlier." ${ }^{15}$ Wolf et al. suggest targeted screening of aging patients. ${ }^{2}$

The issue of screening for melanoma remains controversial to this moment, as illustrated by two publications released in late summer of 2013. Geller et al. report recent statistics from Connecticut, the site of the oldest population-based cancer registry in the United States. ${ }^{16}$ Noting "unremitting increases in incidence and mortality of melanoma," beyond that expected from increased reporting, the authors call for large programs of primary prevention and screening of high-risk populations for melanoma in the United States. Just several days prior, Esserman and colleagues placed melanoma screening in the category of that which "expands the population of indolent tumors, with little or no effect on the small population of more aggressive tumors.” ${ }^{17}$

Guided by these recent narrative reviews and original 
research, let us examine the evidence which would favor some form of screening for melanoma in the primary care setting.

\section{Recent Evidence Regarding Screening}

It has long been known, ${ }^{4,14,15}$ and recently reaffirmed, ${ }^{18}$ that detection of thinner (earlier) melanoma lesions reduces mortality, and if detected early enough, melanoma is potentially curable in all patients. ${ }^{4}$ Swetter et al. studied 566 adult invasive melanoma patients of clinics in California and Michigan using survey methodology. ${ }^{19}$ Thinner tumors $(\leq 1 \mathrm{~mm})$ were associated with younger age, female gender, higher education level, and physician discovery. Physician TBSE was associated with thinner tumors, largely due to the effect of this examination in men over 60 years old. Patients who used a melanoma picture as a visual aid (provided by the study) and performed their own TBSE were more likely to have thinner tumors. The authors concluded that physician and patient TBSE were complementary early detection strategies, particularly in men over 60 . They further suggested that physician TBSE may be the more practical approach for early detection in the high-risk group of older men. ${ }^{19} \mathrm{~A}$ community-based, case-control telephone survey study of 3,762 cases and 3,824 controls in Queensland, Australia, associated TBSE in the three years before diagnosis with a $14 \%$ lower risk of being diagnosed with a thick melanoma $(>0.75 \mathrm{~mm}){ }^{20}$

Additional studies from this area of Australia yield statistics regarding melanoma screening. Data from 16,383 TBSEs of patients in a centrally-organized program of skin screening clinics staffed by trained primary care physicians, with all patients having suspect lesions being referred to their own physician for further treatment, ${ }^{21}$ includes: 1) a total of 2,302 referrals were made for 4,129 suspect lesions, and 2) data from $95 \%$ of the 1,417 lesions which were excised included a yield of 33 melanomas $(0.2 \%$ of all TBSEs), 252 basal cell carcinomas and 97 squamous cell carcinomas. The calculated specificity of TBSE for melanoma was $86 \%$ (which the authors suggest is comparable to that of other screening tests such as mammography). The sensitivity of TBSE for melanoma was not calculated, as follow-up of those who had a negative TBSE was not conducted. ${ }^{21}$ A related study collected data on 28,755 skin examinations (resulting in 11,403 excisions or biopsies) from random samples of family medicine physicians excising or performing biopsy of skin lesions of patients in their routine practice, and from family physicians working in the aforementioned centrally-organized skin screening clinics. $^{22}$ The number (of pigmented lesions) needing to be excised (NNE) to find one melanoma was 19.6, a number that was significantly higher if there was strong patient pressure to excise the lesion, and significantly lower if the physician thought the lesion was likely to be malignant. The authors admit that determining an ideal number needed to excise is difficult given the risk of missing melanoma versus the need to reduce health care costs. ${ }^{22}$ It may be noted that the NNE generally varies from 20-40 for primary care clinicians in non-specialized clinics, and 4-18 for dermatologists at specialty clinics. ${ }^{23} \mathrm{~A}$ multinational, multicenter prospective trial recruited 14,381 subjects from all willing adult patients seeking dermatology consultation for focused skin lesions. As a result, a TBSE was initiated for each participant and suspicious or equivocal lesions were excised or biopsied. Melanoma was detected in 40 subjects $(0.3 \%)$, and nonmelanoma skin cancer in 299 $(2.1 \%) .{ }^{24}$ In this study, 47 patients needed to be examined by TBSE to find one skin malignancy, and 400 patients examined to detect one melanoma. The authors calculated the risk of missing one malignancy if not performing TBSE at $2.2 \%$.

The aforementioned investigations suggest that melanoma screening would likely result in these skin cancers being found at an earlier stage, and provide approximate figures for the sensitivity, specificity and NNE regarding such screening. These studies, like most all such studies in the past, ${ }^{2}$ do not address whether or not these efforts would save lives or be cost effective. A community-based study of melanoma screening was undertaken in a population of 560,000 persons in Queensland, Australia. The study was designed to detect a $20 \%$ reduction in melanoma mortality rates during a 15 -year period. The trial, however, could not be completed due to the significant cost of the program. ${ }^{14,25}$ A compelling, 2003-2004 population-based skin cancer screening project included 360,288 adults 20 years or older in an area of Germany. ${ }^{26}$ Subjects were initially screened by TBSE with a generalist physician. If suspicious lesions were identified, the individual was referred to a dermatologist for a second examination. Trends in melanoma mortality rates in this area were compared to adjacent regions and other parts of Germany. At a 5-year follow-up, the melanoma mortality rate declined by $47 \%$ in men and by $49 \%$ in women in this region of the country, while mortality rates were stable in the other areas. The authors of the update of the USPSTF recommendations point out that an adequately powered, population-based randomized controlled trial of screening in the United States would require approximately 800,000 subjects in order to demonstrate favorable mortality outcomes of screening for skin cancer. ${ }^{12}$ Such a trial is unlikely to be 

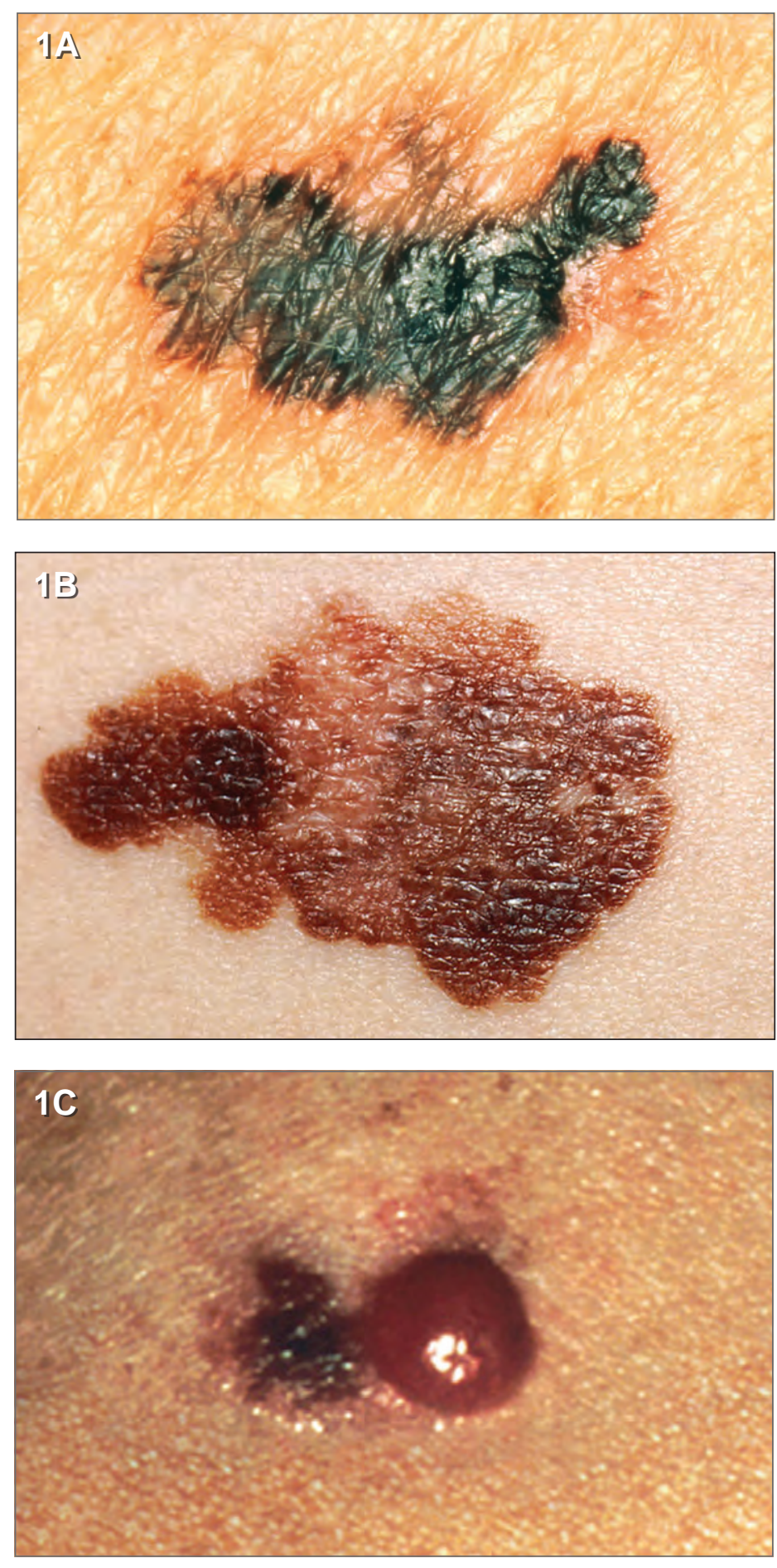

Figure 1. Examples of melanoma lesions. All three lesions exhibit uneven color and irregular borders. [Photographs courtesy of National Cancer Institute (http://www.cancer.gov).]

undertaken given the tremendous cost. Older models of costeffectiveness suggest that screening programs for melanoma may be comparable to that of other cancer screening programs currently in place in the United States in this regard. 2,4,14,15

\section{Screening Methods}

Screening for melanoma during a TBSE by a primary care clinician using the unaided eye generally involves either the use of the ABCDE mnemonic, or the "ugly duckling sign." The ABCDE mnemonic involves systematic examination of lesions for the presence of asymmetry (A), irregular or ill-defined border (B), variation in color from one area of the same lesion to another (C), diameter larger than $6 \mathrm{~mm}$ (D), and if the mole is evolving (changing in size, shape or color) or looks different than surrounding moles (E). The latter statement of the "E" portion of the mnemonic is the concept of the easily taught method of the "ugly duckling sign" which is to identify and investigate the mole which looks different than surrounding moles. In addition, depigmentation, ulceration and bleeding within a melanocytic lesion suggest the presence of malignant melanoma. ${ }^{27}$ For illustration, Figure 1 includes examples of cutaneous melanoma, and Figure 2 presents examples of skin lesions which may resemble melanoma. Melanoma may also occur in the nail apparatus as new pigmented lines on a single nail or dyspigmentation of the nail bed or nailfold. ${ }^{1,27}$ Comprehensive TBSE includes not only skin surfaces, but nail beds, palms, soles and mucocutaneous surfaces (e.g. careful visual inspection of the vulvar and vaginal mucosa during a pelvic examination). The reader is referred to a recent publication, ${ }^{1}$ and a variety of available dermatologic atlases for further details and color pictures to aid in melanoma screening.

Total-body photography may be useful for documentation and for follow-up of patients being screened in the office for melanoma. ${ }^{27}$ The cost-effectiveness of this strategy has not been determined.

One concern is that while Americans make an average of 1-2 visits to primary care physicians each year, these clinicians may not be adequately trained to identify early melanoma. ${ }^{14}$ A recent systematic review revealed that while many specific educational interventions have been developed for primary care physician skin cancer education, including one aimed at medical students on a primary care rotation, ${ }^{28}$ there is variability in the particular interventions and the design to study them. Few have been rigorously evaluated. ${ }^{29}$ Overall rates of TBSE remain low in the United States, and disparities regarding screening remain for Hispanics, nonwhite racial groups and those with less education or lower health literacy. ${ }^{30}$

Dermoscopy (several synonyms) is a hand-held, noninvasive, real-time imaging technique to aid in the detection of skin cancer, including melanoma. It is available, with training, for use by primary care physicians. ${ }^{31}$ In the hands of dermatologists, this technique has been shown to increase 
sensitivity and specificity of skin cancer diagnosis, and decrease the NNE, compared to the naked eye. ${ }^{23}$ Primary care physicians also increase their sensitivity for detecting melanoma with the use of this technology. ${ }^{1,27}$ Argenziano and colleagues have suggested that dermoscopy improves melanoma recognition. Signs of malignancy in melanoma become visible with dermoscopy much sooner than with the naked eye (the ABC portion of the ABCDE criteria become more evident only when the melanoma is relatively large). In addition, clinicians may more closely examine the more benign looking lesions if dermoscopy is available, and use this technique to monitor their patients. ${ }^{23}$

Available dermoscopic techniques include contact or noncontact dermoscopy with or without a liquid interface. Direct contact and the liquid interface provide maximum image clarity. Descriptions of the technical aspects of this procedure are readily available..$^{27,31}$ The reader is referred to the very recent publication of Marghoob et al. for excellent pictorial descriptions. ${ }^{31}$ A large study of dermatologists performing this procedure in pigmented skin lesion clinics revealed that the time needed for TBSE was 70 seconds without dermoscopy and 142 seconds with dermoscopy. ${ }^{32}$

Earlier studies have shown that self-skin examination (SSE), often in conjunction with public educational campaigns, has resulted in earlier presentation and thinner melanoma lesions upon presentation. ${ }^{14,15,19}$ In the aforementioned study by Swetter et al., most melanoma lesions were discovered by SSE, rather than a physician, with important contributions by family and friends. Thinner lesions, however, were associated with physician diagnosis. ${ }^{19}$ In that study, a combination of regular SSE and a melanoma visual aid were associated with thinner tumors. A randomized trial of Rhode Island and Massachusetts residents from 2000 to 2001, observed a $49 \%$ increase in SSE at one year in the intervention group. Helpful interventions included a video, a hand mirror, a shower card, brochures and sample photographs, as well as a health educator. ${ }^{33}$ In an Australian study of men over 50, the addition of a video or DVD to written materials had only a transient advantage for optimization of SSE. ${ }^{34}$

Recently, new technology for patient-assisted melanoma detection has become available to consumers. Dermoscope attachments are now available for mobile telephones, allowing the consumer to send images electronically to a teledermatologist. The feasibility of this technology was demonstrated in 10 patients in Australia. ${ }^{35}$ Currently these items are available for approximately $\$ 450$. No outcomes data regarding their use has been published. A number of other

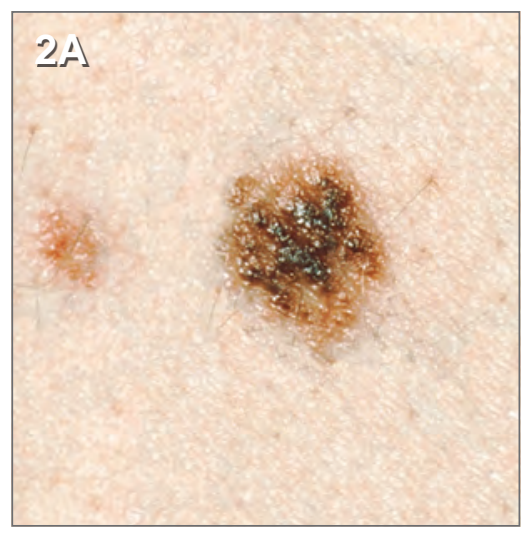

Figure 2.

Nonmalignant pigmented skin lesions variably resembling melanoma. Lesion $2 \mathrm{~A}$ is a dysplastic nevus. Lesions $2 B$ and $2 \mathrm{C}$ are common moles. [Photographs courtesy of National Cancer Institute (http://www.cancer.gov).]
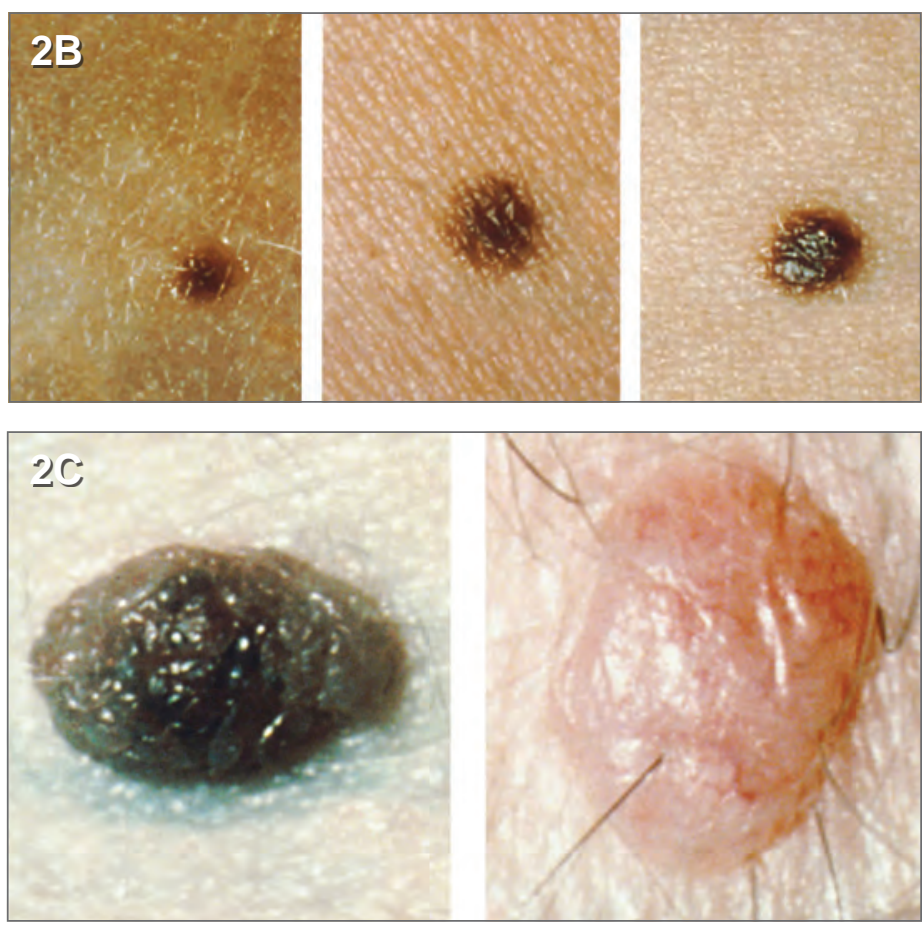

smart phone applications are available. The performance of the software appears to be highly variable. ${ }^{36}$

\section{A Few Words Regarding Biopsy}

Any suspicious lesion encountered by a primary care clinician should be biopsied, after carefully documenting the appearance and the dimensions of the lesion in the medical record. ${ }^{1}$ The preferred method of excision for diagnosis of a lesion suspected to be melanoma involves complete removal of the lesion, generally with the use of an elliptical incision made with a scalpel. Margins of normal-appearing skin should be 1-3 mm peripheral to the lesion, and a deep margin of subcutaneous fat, including appendageal structures. Punch biopsies for excision may also be used if the entire pigmented lesion, with margin, will fit entirely within the trephine. ${ }^{27}$ While use of deep scoop or shave biopsies (saucerization) may be appropriate in some instances, the use of superficial shave biopsies are not recommended when invasive melanoma is suspected. ${ }^{1,27}$ The reader is referred to 
the excellent review by Tran et al., for details regarding the biopsy of pigmented lesions, including those of the nails. ${ }^{27}$

\section{Community Empowerment}

Finally, an additional important strategy is for primary care clinicians to empower their panel of patients to prevent and help diagnose melanoma. Mitchell and Leslie, in their recent review, ${ }^{15}$ suggest that primary prevention messages (principally focused on sun avoidance) have been an overemphasized component of melanoma outreach education. While not recommending abandonment of such efforts, they point out that even in intensive campaigns, long-term behavioral changes have been very difficult to sustain. Primary care clinicians may be able to make an impact by inducing their high risk patients to have "skin-awareness." Patients may be referred to the ACS website as it contains excellent information for patients, including instructions on SSE: http://www.cancer.org/acs/groups/cid/documents/ webcontent/003184-pdf.pdf.

While more than half of all melanomas may be detected by patients, those individuals at highest risk for melanoma may be those least likely to seek skin cancer screening. ${ }^{2}$ For example, women are more likely to detect melanomas in both themselves and their spouses. Yet some high-risk individuals do apparently recognize their personal risk factors and notice suspicious lesions. ${ }^{4}$ For several years it has been suggested that educational outreach to encourage SSE for melanoma be targeted toward middle-aged and older men, particularly those with changing moles or skin types I and II (those who always or usually sunburn). ${ }^{4,15}$ In addition, some suggest that any clinician TBSE include SSE education. ${ }^{15}$ The latter authors point out that the USPSTF does not recommend routine physician or patient breast examination, yet a significant majority of both groups of individuals perform them. ${ }^{15}$ They further herald "causerelated marketing” such as breast cancer awareness, and basically suggest the need for a slogan and a symbol to aid in melanoma outreach education to high-risk individuals.

\section{Synthesis}

As discussed above, definitive clinical outcomes and cost effectiveness studies regarding the wisdom of screening populations of patients in the United States for melanoma may never be forthcoming. Once the examination room door closes, however, the overall health and well-being of that particular patient is of immediate importance to the practicing primary care clinician. Lack of adequate time with patients at office encounters is a perceived barrier to provision of "high-quality medical care" in the primary care setting, ${ }^{37}$ and this situation is not likely to improve. The successful provision of preventive screenings and education during a primary care office visit is complex and includes patient, physician, relationship and visit contextual factors, such as the number of problems brought to the fore by the patient at that encounter. ${ }^{38}$ As seen above, TBSE at a routine office visit likely adds 70 or more seconds, ${ }^{32}$ and the education associated with it perhaps another minute. The challenge for the primary care clinician, given the constraints of limited office visit time and resources, is to effectively and individually prioritize interventions and screenings that would most improve the future health of a particular patient at that time.

It would seem prudent, based on what we have learned, to empower patients to have "skin awareness" and report suspicious findings, and to perform TBSE and teach SSE to high-risk patients. The latter would include middle-aged and older men, persons of all ages who are at risk due to skin type and behavior such as the use of tanning beds, those with a first-degree relative with melanoma, and other individuals at risk or who are concerned about their skin. It may also be appropriate, based on the clinician's relationship with a particular patient and perceived receptivity to advice, to offer primary skin protection such as sun avoidance. An algorithm based on these considerations is found in Figure 3.

\section{Conclusion}

Cutaneous malignant melanoma incidence is increasing in the United States. It is associated with significant morbidity and mortality, but is potentially curable if found at an early stage. Despite lack of recommendation from the USPSTF, and current controversy, recent evidence has suggested that primary care clinicians, who are entrusted with the overall health and well-being of their patients, strongly consider targeted in-office screening, and education regarding home skin surveillance, of their high-risk patients. All suspicious lesions should be subjected to adequate biopsy.

\section{Conflicts of Interest}

None.

\section{References}

1 Shenenberger DW. Cutaneous malignant melanoma: a primary care perspective. Am Fam Physician. 2012;85:161-168.

2 Wolf J, Harris R, Ferris LK. Screening for melanoma in aging patients. Cutis. 2013;91:81-86.

3 Little EG, Eide MJ. Update on the current state of melanoma incidence. Dermatol Clin. 2012;30:355-361.

4 Geller AC, Sober AJ, Zhang Z, et al. Strategies for improving melanoma education and screening for men age $\geq 50$ years. Cancer. 2002;95:1554-61. 
Figure 3. Suggested Algorithm for Primary Care Office Screening and Prevention of Cutaneous Malignant Melanoma

\section{General Counseling Regarding UV Radiation Avoidance and Protection and Skin Awareness}

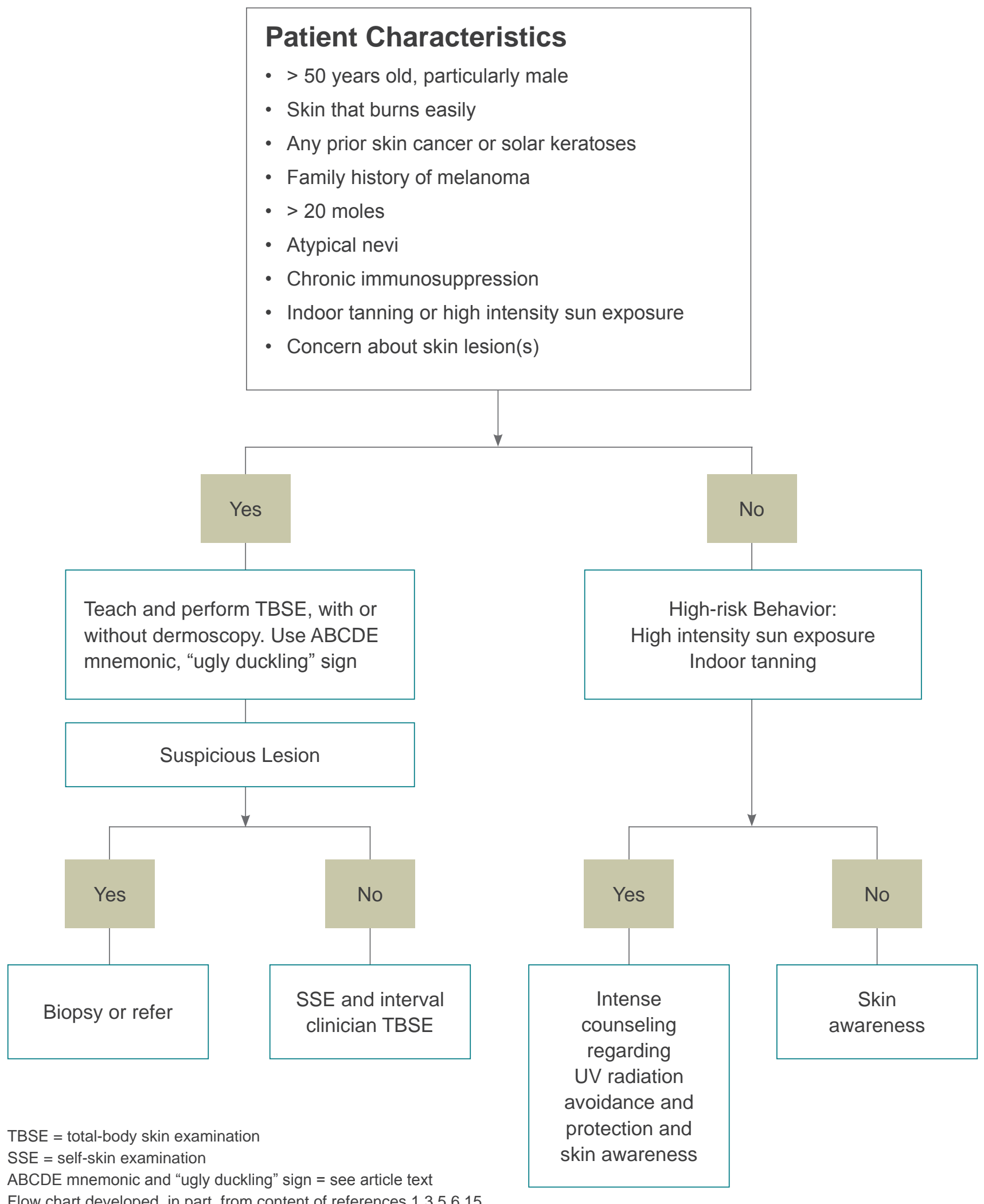

Flow chart developed, in part, from content of references 1,3,5,6,15 
5 Russak JE, Rigel DS. Risk factors for the development of primary cutaneous melanoma. Dermatol Clin. 2012;30:363-368.

6 Psaty EL, Scope A, Halpern AC, Marghoob AA. Defining the patient at high risk for melanoma. Int J Dermatol. 2010;49:362-376.

7 Fogel J, Krausz F. Watching reality television beauty shows is associated with tanning lamp use and outdoor tanning among college students. J Am Acad Dermatol. 2013;68:784-789.

8 Richards TB, Johnson CJ, Tatalovich Z, et al. Association between cutaneous melanoma incidence rates among white US residents and county-level estimates of solar ultraviolet exposure. J Am Acad Dermatol. 2011;65:S50.e1-9.

9 Baade P, Meng X, Youlden D, Aitken J, Youl P. Time trends and latitude differences in melanoma thickness distribution in Australia, 1990-2006. Int J Cancer. 2012;130:170-178.

10 Cokkinides VE, Geller AC, Jemal A. Trends in melanoma mortality among non-Hispanic whites by educational attainment, 1993-2007. Arch Dermatol. 2012;148:587-591.

11 US Preventive Services Task Force. Screening for skin cancer: recommendations and rationale. Am J Prev Med. 2001;20:44-46.

12 Wolff T, Tai E, Miller T. Screening for skin cancer: an update of the evidence for the U.S. Preventive Services Task Force. Ann Intern Med. 2009;150:194-198.

13 Berwick M, Armstrong BK, Ben-Porat L, et al. Sun Exposure and mortality from melanoma. J Natl Cancer Inst. 2005;97:195-199.

14 Curiel-Lewandrowski C, Chen SC, Swetter SM. Screening and prevention measures for melanoma: is there a survival advantage? Curr Oncol Rep. 2012;14:458-467.

15 Mitchell JK, Leslie KS. Melanoma death prevention: moving away from the sun. J Am Acad Dermatol. 2013;68:e169-179.

16 Geller AC, Clapp RW, Sober AJ, et al. Melanoma epidemic: an analysis of six decades of data from the Connecticut Tumor Registry. J Clin Oncol. 2013;31: on-line ahead of print, DOI: 10.1200/ JCO.2012.47.3728.

17 Esserman LJ, Thompson IM Jr., Reid B. Overdiagnosis and overtreatment in cancer: an opportunity for improvement. JAMA. 2013;310:797-798.

18 Balch CM, Gershenwald JE, Soong SJ, et al. Final version of 2009 AJCC melanoma staging and classification. J Clin Oncol. 2009;27:6199-6206.

19 Swetter SM, Pollitt RA, Johnson TM, Brooks DR, Geller AC. Behavioral determinants of successful early melanoma detection. Cancer. 2012;118:3725-3734.

20 Aitken JF, Elwood M, Baade PD, Youl P, English D. Clinical wholebody skin examination reduces the incidence of thick melanomas. Int J Cancer. 2010;126:450-458.

21 Aitken JF, Janda M, Elwood M, et al. Clinical outcomes from skin screening clinics within a community-based melanoma screening program. J Am Acad Dermatol. 2006;54:105-14.

22 Baade PD, Youl PH, Janda M, et al. Factors associated with the number of lesions excised for each skin cancer. Arch Dermatol. 2008;144:1468-1476.

23 Argenziano G, Albertini G, Castagnetti F, et al. Early diagnosis of melanoma: what is the impact of dermoscopy? Dermatol Ther. 2012;25:403-409.

24 Argenziano G, Zalaudek I, Hofmann-Wellenhof R, et al. Total body skin examination for skin cancer screening in patients with focused symptoms. J Am Acad Dermatol. 2012;66:212-219.

25 Janda M, Lowe JB, Elwood M, et al. Do centralized skin screening clinics increase participation in melanoma screening (Australia)? Cancer Causes Control. 2006;17:161-168.

26 Katalinic A, Waldmann A, Weinstock MA, et al. Does skin cancer screening save lives? Cancer. 2012;118:5395-5402.
27 Tran KT, Wright NA, Cockerell CJ. Biopsy of the pigmented lesion when and how. J Am Acad Dermatol. 2008;59:852-871.

28 Jain N, Anderson MJ, Patel P, et al. Melanoma simulation model: promoting opportunistic screening and patient counseling. JAMA Dermatol. 2013;149:710-716.

29 Goulart JM, Quigley EA, Dusza S, et al. Skin cancer education for primary care physicians: a systematic review of published evaluated interventions. J Gen Intern Med. 2011;26:1027-1035.

30 Amrock SM, Meydani A. Trends and disparities in total-body skin examination: evaluating the National Health Interview Survey, 20002010. JAMA Dermatol. 2013;149:363-364.

31 Marghoob AA, Usatine RP, Jaimes N. Dermoscopy for the family physician. Am Fam Physician. 2013;88:441-450.

32 Zalaudek I, Kittler H, Marghoob AA, et al. Time required for a complete skin examination with and without dermoscopy: a prospective, randomized multicenter study. Arch Dermatol. 2008;144:509-513.

33 Lee KB, Weinstock MA, Risica PM. Components of a successful intervention for monthly skin self-examination for early detection of melanoma: the "check it out" trial. J Am Acad Dermatol. 2008;58:1006-1012.

34 Janda M, Neale RE, Youl P, et al. Impact of a video-based intervention to improve the prevalence of skin self-examination in men 50 years or older. Arch Dermatol. 2011;147:799-806.

35 Janda M, Loescher LJ, Soyer HP. Enhanced skin self-examination: a novel approach to skin cancer monitoring and follow-up. JAMA Dermatol. 2013;149:231-236.

36 Wolf JA, Moreau JF, Akilov O, et al. Diagnostic inaccuracy of smartphone applications for melanoma detection. JAMA Dermatol. 2013;149:422-426.

37 Deshpande SP, DeMello J. A comparative analysis of factors that hinder primary care physicians' and specialty physicians' ability to provide high-quality care. Health Care Manag. 2011;30:172-178.

38 Shires DA, Stange KC, Divine G, et al. Prioritization of evidencebased preventive health services during periodic health examinations. Am J Prev Med. 2012;42:164-173.

(C) 2014 Aurora Health Care, Inc. 